Anaesthesist

$2001 \cdot 50: 46-51 \odot$ Springer-Verlag 2001

Redaktion

R. Larsen, Homburg/Saar
Intensivmedizin

H. Lode ${ }^{1} \cdot$ R. Erbes ${ }^{1} \cdot$ H. Geerdes-Fenge ${ }^{1} \cdot$ M. Raffenberg ${ }^{1} \cdot$ H. Mauch ${ }^{2}$

${ }^{1}$ Abteilung für Pneumologie und Infektiologie, Klinik Heckeshorn, Akademisches Lehrkrankenhaus der Freien Universität Berlin

${ }^{2}$ Medizinische Mikrobiologie, Klinik Heckeshorn, Akademisches Lehrkrankenhaus

der Freien Universität Berlin

\section{Nosokomiale Pneumonie}

\section{Epidemiologie, Pathogenese und Diagnostik ${ }^{*}$}

\section{Zusammenfassung}

Diese Arbeit gibt eine Übersicht über die aktuellen epidemiologischen, pathogenetischen, ätiologischen und diagnostischen Erkenntnisse bei der nosokomialen Pneumonie. Diese Erkrankung steht in den westlichen Industrieländern an zweiter Stelle der nosokomialen Infektionen, bei Intensivpatienten ist insbesondere die Beatmungspneumonie die dominierende Infektion. In der Pathogenese spielen die Kolonisation des Oropharynx und nachfolgend der Trachea mit gramnegativen Keimen eine dominierende Rolle und bestimmen deshalb auch die Erregerverteilung der nosokomialen Pneumonie. Hierbei hat es sich bewährt, die frühe Manifestation einer nosokomialen Pneumonie mit eher einfach zu behandelnden Erregern von der Erregerpopulation der späten Pneumonie nach Tag 5 bis 7 im Krankenhaus mit deutlich größeren Resistenzproblemen zu unterscheiden. In der Diagnostik gibt es bisher keinen absolut zuverlässigen Standard; die Bedeutung der bronchoskopischen Verfahren wird dargestellt und die Relevanz ihrer Ergebnisse diskutiert.

\section{Schlüsselwörter}

Nosokomiale Pneumonie - Epidemiologie · Pathogenese · Erregerspektrum · Diagnostik ie nosokomiale Pneumonie ist die zweithäufigste nosokomiale Infektion in den westlichen Industrieländern und zusammen mit der Sepsis die führende Ursache für einen infektionsbedingten Tod im Krankenhaus [3]. Insbesondere auf Intensivstationen bei beatmeten Patienten liegt die Inzidenz einer nosokomialen Pneumonie zwischen 9-68\% und die Letalität bewegt sich zwischen $33 \%$ und $71 \%$ [28]. In 2 epidemiologischen Studien zur Erfassung von Intensivstationsinfektionen sowohl in Europa als auch in den USA waren Pneumonien die dominierenden nosokomialen Infektionen bei intensivmedizinisch betreuten Patienten ([4, 31]; (Tabelle 1). Trotz ihrer Häufigkeit und des ungünstigen Verlaufes sind noch viele diagnostische, pathogenetische und therapeutische Probleme ungeklärt. In der nachfolgenden Übersicht sollen neuere Aspekte und Befunde hinsichtlich der Epidemiologie, der Pathogenese und der Diagnostik der nosokomialen Pneumonie insbesondere bei Intensivpatienten dargestellt werden.

\section{Epidemiologie}

Patienten in Intensivstationen entwickeln im Vergleich zu Patienten auf Normalstationen in einem hohen Prozentsatz nosokomiale Infektionen mit daraus resultierenden längeren Krankenhausverweildauern und entsprechend höheren Kosten. In einer Analyse vom CDC in Atlanta, USA, wurde die Rate der nosokomialen Infektionen bei Erwachsenen auf medizinischen Intensivstationen auf der Basis von standardisierten Definitions- kriterien prospektiv untersucht [24]. Insgesamt 181.993 Patienten in 112 internistischen Intensivstationen wurden zwischen Januar 1992 und Juli 1997 erfasst. Harnwegsinfektionen traten am häufigsten (31\%) auf, gefolgt von Pneumonien $(27 \%)$ und primären Bakteriämien (19\%). Etwa 86\% der registrierten nosokomialen Pneumonien waren mit einer Beatmung assoziiert. Die häufigsten Erreger waren gram-negative aerobe Keime, unter denen Pseudomonas aeruginosa mit $21 \%$ dominierte.

In Deutschland wurde in der NIDEP-Studie bei insgesamt 3,5\% nosokomialen Infektionen in 72 Krankenhäusern die tiefe Atemwegsinfektion mit $20,6 \%$ an zweithäufigster Position ermittelt [17].

Sehr nützliche epidemiologische Informationen wurden auch in einer umfangreichen multizentrischen randomisierten doppelblinden Studie in Kanada generiert, in der zwischen 1992 und 1994 insgesamt 1200 beatmete Patienten prospektiv untersucht wurden. Das eigentliche Studienziel war der Vergleich einer Ulkusprophylaxe mit Sucralfat vs. Ranitidin [6]. Aus unterschiedlichen Gründen mussten 186 Patienten von der abschließenden Analyse ausgeschlossen werden, so dass insgesamt 1014 Patienten hinsichtlich der Risikofaktoren für

*Erstpublikation in Der Internist (2000) 41:1170-1179

Prof.Dr.H.Lode

Abteilung Pneumologie I, Klinik Heckeshorn, Zum Heckeshorn 33, 14109 Berlin 
Tabelle 1

Infektionen auf Intensivstationen: Eintagesprävalenzstudien. (Nach [4, 31])

\begin{tabular}{lcc} 
& USA 1994 & Europa 1992 \\
\hline Anzahl Krankenhäuser (ITS) & 118 & 1417 \\
Anzahl Patienten & 1740 & 10038 \\
Anteil nosokomialer Infektionen [\%] & 25 & 30,3 \\
Infektionen [\%] & & \\
- Pneumonien & 37 & 46,9 \\
- Urogenitale Infektionen & 18 & 17,6 \\
- Sepsis & 14 & 6,9 \\
- Wundinfektionen & 9,4 & 30 \\
Anteil Patienten mit Chemotherapie [\%] & 61 & 62,30
\end{tabular}

eine Beatmungspneumonie bewertet werden konnten. Die Definition der Pneumonie erfolgte auf der Basis unterschiedlicher Kriterien, wobei sowohl klinische als auch mikrobiologische quantitative Parameter herangezogen wurden. Eine Beatmungspneumonie entwickelten demnach 177 Patienten (17,5\%) im Median 7 Tage nach Aufnahme auf die Intensivstation. Unabhängige Risikofaktoren in der Multivarianzanalyse waren die primäre Aufnahmediagnose einer Verbrennung (OR 5,09), eines Polytrauma (OR 5,00), einer ZNS-Erkrankung (OR 3,40) sowie pulmonaler (OR $2,79)$ oder kardialer Vorerkrankungen (OR 2,72; [7]).

In der gleichen kanadischen Studie wurde auch die additive Morbidität und Letalität der Beatmungspneumonie analysiert [18]. Im Vergleich zu den Kontrollpatienten ohne eine Beatmungspneumonie verlängerte sich bei den Pneumoniepatienten der Intensivaufenthalt um im Mittel 4,3 Tage und das relative Risiko für einen letalen Verlauf stieg um 32,3\% an. Insbesondere bei Infektionen durch sogenannte Risikoerreger mit hoher Resistenz verlängerte sich die Liegedauer auf der Intensivstation auf im Mittel 9,1 Tage, im Vergleich zu nur 2,9 Tage bei Infektionen durch sogenannte einfach zu behandelnde Erreger.

Eine niedrigere Inzidenz von $7,8 \%$ Beatmungspneumonien wurde in einer prospektiven Studie in 2 Universitätskliniken in Memphis (USA) beobachtet [16]. Die Diagnose der Pneumonie wurde auf quantitative bronchoskopische Techniken (geschützte Bürste/BAL) basiert, um eine falsche Klassifikation $\mathrm{zu}$ vermeiden. Unter den 358 prospektiv er- fassten Patienten wurden 28 Pneumonien beobachtet, was einer Inzidenz von 12,5 Infektionen auf 1000 Patiententage und 20,5 Pneumonien auf 1000 Beatmungstage entsprach. Mittels multipler logihängige Risikofaktoren für die Entwicklung einer Beatmungspneumonie folgende Faktoren ermittelt werden: eine Serumalbuminkonzentration zum Zeitpunkt der Aufnahme unter 2,2 g/dl (OR 5,9), ein maximaler PEEP von mehr als $7,5 \mathrm{~cm} \mathrm{H}_{2} \mathrm{O}(\mathrm{OR} 4,6)$, eine nicht vorhandene Antibiotikatherapie (OR 6,7 ), die Kolonisation der oberen Atemwege mit gram-negativen respiratorischen Erregern (OR 3,4), eine Raucheranamnese (OR 2,3 für jeweils 50 stischer Regression konnten als unab-
Packungsjahre) und die Dauer der kontrollierten Beatmung (OR 3,4 für $14 \mathrm{Be}$ atmungstage).

\section{Ätiologie und Pathogenese}

Die oropharyngeale Flora des hospitalisierten Patienten ist zumeist der Ausgangsherd für tiefe Atemwegsinfektionen. Kolonisierende Erreger in der Trachea und der Lunge stammen sehr selten aus einer hämatogenen Streuung, sondern sind zumeist über Mikroaspiration aus dem Oropharynx in die tiefen Atemwege gelangt $[9,12]$. Bis heute ist nicht komplett bekannt, warum der Oropharynx im Hospitalbereich schnell mit gram-negativen Erregern kolonisiert wird. Ein bedeutsamer Faktor könnte Stress darstellen, der zur Produktion von Enzymen beiträgt, welche protektive Rezeptoren an der Oberfläche der oropharyngealen Schleimhautzellen schädigen. Diese Veränderungen fördern die Adhäsion von gram-negativen Erregern [19].

In einer neueren prospektiven Studie aus Johannesburg (Südafrika) wurde der Umfang und die Sequenz der Kolonisation von endotrachealen Tuben bei Patienten unter Beatmung untersucht [14]. Im ersten Teil der Studie mit $21 \mathrm{~Pa}$ tienten wurde nach der Extubation festgestellt, dass das untere Drittel des Beatmungstubus durchweg mit einem Sekretfilm bedeckt war, der mittels elek-

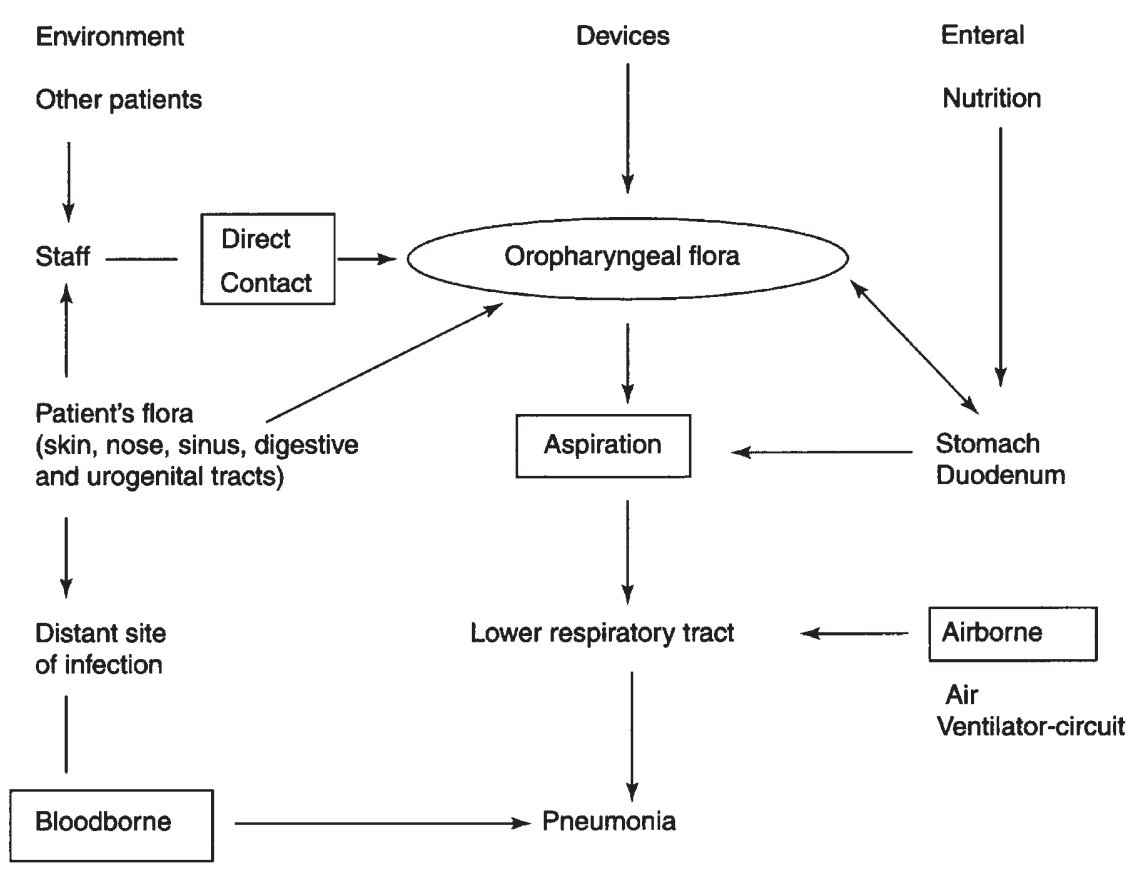

Abb. $1 \Delta$ Infektionswege bei der Beatmungspneumonie. (Nach [15]) 
Tabelle 2

Erreger der Beatmungspneumonien. (Nach [15])

\begin{tabular}{|c|c|c|}
\hline Frühe Pneumonien ( $\leq 5$ Tage) & Späte Pneumonien (>5 Tage) & Andere Erreger \\
\hline Streptococcus pneumoniae & Pseudomonas aeruginosa & Anaerobier \\
\hline Haemophilus influenzae & Enterobacter spp. & Legionella pneumophila \\
\hline Moraxella catarrhalis & Acinetobacter spp. & Influenza $A$ und $B$ \\
\hline Staphylococcus aureus & Klebsiella pneumoniae & RS-Virus \\
\hline Aerobe gram-negative Erreger & $\begin{array}{l}\text { Serratia marcescens } \\
\text { Escherichia coli } \\
\text { Andere gram-negative Erreger } \\
\text { Staphylococcus aureus }\end{array}$ & Pilze \\
\hline
\end{tabular}

tronenmikroskopischem Scan als Biofilm identifiziert werden konnte. Aus dieser Oberflächenschicht konnten bei 18 Patienten gram-negative Erreger isoliert werden. Im zweiten Teil der Studie mit 10 Patienten wurde die Sequenz der Kolonisation exakt bestimmt. Nach der Intubation wurde der Oropharynx innerhalb von $36 \mathrm{~h}$ kolonisiert, der Magen innerhalb von 36-6o h, die tiefen Atemwege innerhalb von 60-84 h und danach der endotracheale Tubus innerhalb von 60-96 h. Eine nosokomiale Pneumonie im gesamten Patientengut von 31 Patienten entwickelte sich bei 13 beatmeten $\mathrm{Pa}$ tienten. Bei 8 dieser Pneumoniepatienten konnten die identischen Erreger sowohl in den Sekreten der tiefen Atemwege, wie auch im Biofilm des endotrachealen Tubus isoliert werden.

Wie in der Abb. 1 dargestellt, wurde bisher auch der Magen als Reservoir für kolonisierende Erreger in dem pathogenetischen Modell der Pneumonieentstehung beträchtlich betont $[15,23]$. Hierzu korrelierten einige Studien, die im Rahmen der Stressulkusprophylaxe bei beatmeten Patienten mit Sucralfat eine verminderte Rate an nosokomialen Pneumonien fanden und dieses auf die geringeren Keimzahlen unter Sucralfat im Magen bei niedrigerem $\mathrm{pH}$ Wert zurückführten [15]. Diese Auffassungen werden durch 2 kürzlich publizierte Studien nicht bestätigt, in denen zwischen Sucralfat und Antazida bei wirksamer Stressulkusprophylaxe keine Unterschiede hinsichtlich der Pneumoniefrequenz nachgewiesen werden konnten $[7,11]$. Auch die Letalität und die Frequenz der gastrointestinalen Blutung unterschieden sich in diesen Studien bei einer Prophylaxe mit
Sucralfat vs. Ranitidin i.v. nicht wesentlich.

Auch die horizontale Lagerung des beatmeten Patienten kann einen Risikofaktor für die Entwicklung einer nosokomialen Pneumonie darstellen. Kürzlich wurde in einer Studie aus Barcelona erneut gesichert, dass die horizontale Lagerung des Beatmungspatienten einen signifikanten Risikofaktor für eine Beatmungspneumonie darstellt [10]. Bei 86 Patienten, die während einer Beatmung entweder halb hoch (39 Patienten) oder horizontal (47 Patienten) gelagert waren, wurde die Inzidenz einer Beatmungspneumonie festgestellt. Der Unterschied war mit einer Inzidenz von $8 \%$ vs. $34 \%$ zugunsten der erhöhten Lagerung eindeutig.

Hinsichtlich der mikrobiellen Ätiologie hat sich heute weitgehend die Einteilung in die Erreger der sogenannten frühen Pneumonie im Vergleich zu den Erregern der späten Pneumonie durchgesetzt $([1,15]$; (Tabelle 2$)$. Dabei wird davon ausgegangen, dass innerhalb der ersten 4-5 Tage nach Krankenhausaufnahme noch eine weitgehend normale oropharyngeale Flora vorliegt. Erst nach diesem Zeitraum mit verlängerter Hospitalisierung und speziellen Risikofaktoren kommt es zur oropharyngealen Besiedlung mit Enterobacteriaceae und Pseudomonas aeruginosa. Diese zeitliche Zuordnung des Erregermaterials sollte bei der empirischen Behandlung einer Beatmungspneumonie berücksichtigt werden [20].

\section{Diagnostik}

Die Diagnose einer nosokomialen Pneumonie beruht auf dem Nachweis eines neuen und progredienten pulmonalen Infiltrates, Fieber, Leukozytose, CRP-Erhöhung und einem purulenten tracheobronchialen Sekret. Keiner dieser Befunde ist jedoch ausreichend spezifisch und sensitiv [5, 21].

Die Unzulänglichkeit der alleinigen klinischen Diagnose hat in den letzten Jahren dazu geführt, dass quantitative mikrobiologische Analysen des endotrachealen Sekretes oder des bronchoskopisch gewonnenen Materials über eine bronchoalveoläre Lavage (BAL) oder eine geschützte Bürste die Diagnosestellung deutlich präziser gestalten konnten $[5,22,29]$. Am einfachsten zugänglich ist das endotracheale Sekret, welches ohne bronchoskopische Maßnahmen gewonnen werden kann. Cook und Mandell [8] haben kürzlich den Stellenwert der quantitativen Analyse des endotrachealen Sekretes in einer Literaturübersicht gewürdigt. Diese Autoren kommen zu dem Resultat unter Analyse von 12 relevanten Studien, dass die Sensitivität (38\%-100\%) und Spezifität (14\%-100\%) der Ergebnisse des endotrachealen Sekretes erheblich schwanken. Erschwerend kommt bei der Bewertung der Mikrobiologie hinzu, dass eine eindeutige Grenzwertbestimmung von $10^{5}$ oder $10^{6}$ Keime pro ml Sekret bis heute nicht erfolgt ist. Die Analyse des endotrachealen Sekretes kann daher zur Zeit nicht als diagnostisch valide empfohlen werden und weitere gut geplante Studien sind zur weiteren Klärung notwendig.

Zahlreiche Untersuchungen zwischen 1984 und 1995 haben den Stellenwert der semiquantitativen kulturellen mikrobiologischen Analyse der geschützten, bronchoskopisch zu applizierenden Bürste (PSB) versucht zu bestimmen. Eine Würdigung dieser Studien [2] kommt zu dem Ergebnis, dass die Sensitivität dieser Methode zwischen 33 und $100 \%$ schwankt und die Spezifität von 50\%-100\% reicht. Insgesamt erscheint die PSB eher mehr spezifisch als sensitiv in der Diagnose einer Beatmungspneumonie zu sein.

Die quantitative bronchoalveoläre Lavage ist von zahlreichen Autoren als die beste und preisgünstigste Methode zur Diagnostik der nosokomialen Pneumonie dargestellt worden [21]. Allerdings besteht keine Übereinstimmung hinsichtlich der signifikanten Keimzahl (10 4 oder $10^{5}$ Keime pro $\mathrm{ml}$ ) und auch die mäßige Spezifität der Methode bei Pati- 


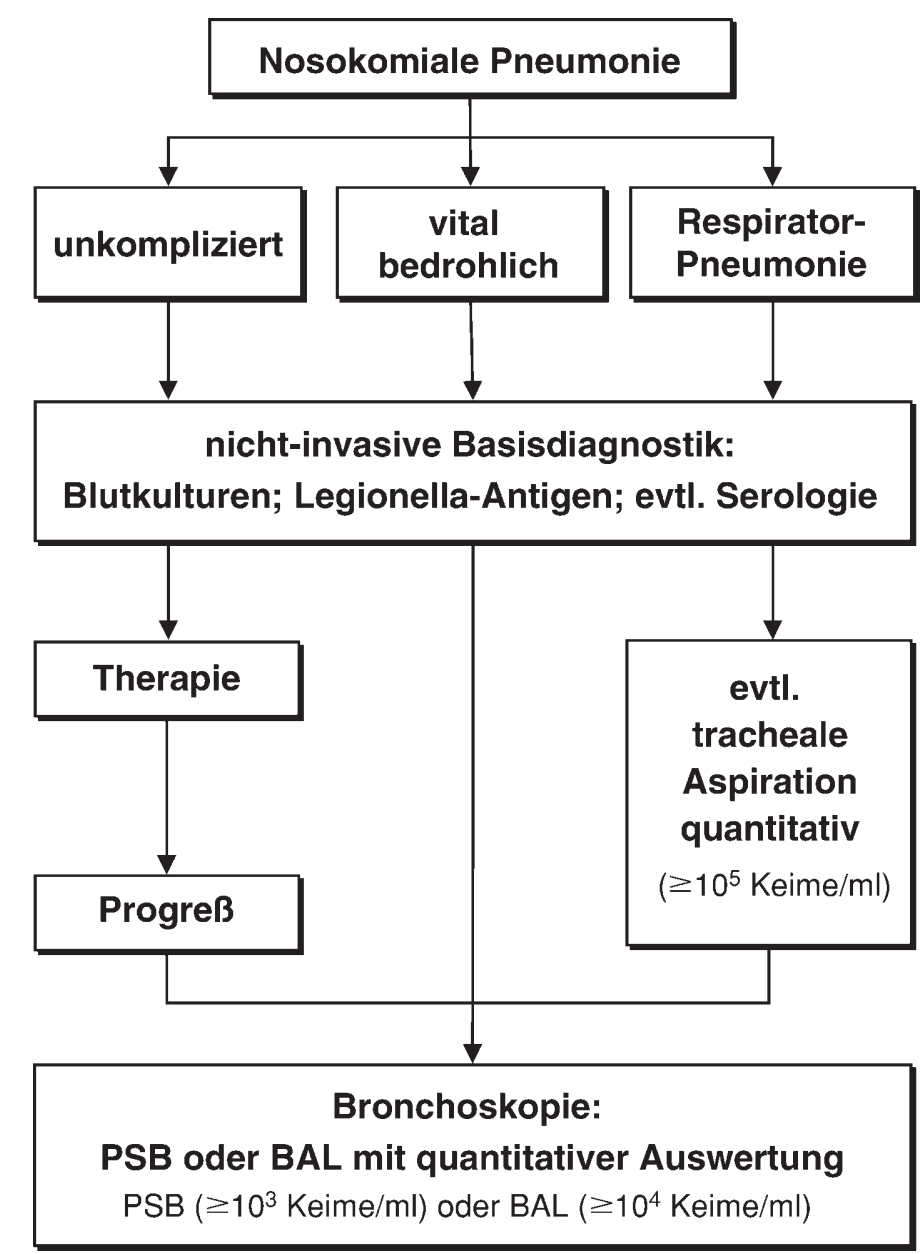

Abb. $2<$ Diagnostik bei nosokomialer Pneumonie enten mit umfangreicher tracheobronchialer Kolonisation wird negativ bewertet. In einer kürzlichen Analyse [30] von 23 seit 1988 publizierten Studien beschreiben die Autoren eine Sensitivität der quantitativen BAL zwischen 42\%-93\% mit im Mittel 73\%. Die Spezifität wird mit im Mittel $82 \%$ angegeben, woraus eine inkorrekte Diagnose bei etwa $20 \%$ der Patienten resultiert. Eine zusätzliche wesentliche Information kann in der BAL durch die mikroskopische Analyse von intrazellulären Erregern in den Alveolarmakrophagen gewonnen werden.

Umstritten ist der Stellenwert der bronchoskopischen Verfahren ebenfalls bei Patienten, die unter einer vorangehenden oder noch laufenden antibiotischen Behandlung stehen. In einer Analyse dieses Problems wurden in Paris 63 Patienten prospektiv untersucht [27]. Unter Berücksichtigung einer vorangegangenen antibiotischen Therapie wurden 3 Patientengruppen gebildet: 12 Patienten ohne Antibiotikatherapie, 31 Pati- enten mit einer laufenden Antibiotikabehandlung (Antibiotikagabe mindestens schon $72 \mathrm{~h}$ vor der Bronchoskopie begonnen) und eine 3. Gruppe mit einer innerhalb von $24 \mathrm{~h}$ vor der Bronchoskopie eingeleiteten antibiotischen Gabe.

Bei 35 Patienten konnte die Diagnose einer Beatmungspneumonie gesichert werden unter Bezug auf histologische Befunde $(n=2)$, Entwicklung eines Abszesses $(n=2)$, positiven Blutkulturen $(n=4)$ oder der Beseitigung eines pulmonalen Infiltrates unter einer adäquaten Antibiotikatherapie $(n=27)$. Für die positiven Sensitivitätsparameter von 5\% intrazellulär nachgewiesenen Erregern in den BAL-Zellen, einer PSB-Kultur von mindestens $10^{3}$ Erregern pro $\mathrm{ml}$ sowie einer positiven BAL-Kultur von $10^{5}$ Keimen pro $\mathrm{ml}$ ergaben sich folgende Ergebnisse: $71 \%, 88 \%$ und $71 \%(\mathrm{~Pa}-$ tientengruppe ohne antibiotische Therapie); $50 \%, 77 \%$ und $83 \%$ für die Patienten unter einer laufenden antibiotischen Behandlung und $67 \%, 40 \%$ und $38 \%$ für die Gruppe mit der kürzlich be- gonnenen antibiotischen Behandlung. Die Spezifität lag durchweg über 90\%.Eine bronchoskopische Analyse mit quantitativer Mikrobiologie erscheint daher auch unter antibiotischer Therapie grundsätzlich möglich - eine Antibiotikapause von $24 \mathrm{~h}$ ist jedoch empfehlenswert.

\section{Stellenwert der invasiven bronchoskopischen Diagnostik}

In den letzten Jahren ist eine beträchtliche Diskussion entstanden, wieweit die invasiven bronchoskopischen Verfahren mit der Erhebung quantitativer mikrobiologischer Analysen einen positiven Einfluss auf den Verlauf einer Beatmungspneumonie haben. In einer offenen prospektiven randomisierten Pilotstudie bei 51 Patienten unter Beatmung über mehr als 72 h wurde dieses Problem untersucht [25]. Die Patienten wurden randomisiert 2 Gruppen zugeteilt: Die Gruppe A mit 24 Patienten wurde einer quantitativen endotrachealen Aspiration unterzogen sowie auch quantitativen Kulturen von PSB und BAL. Bei der Gruppe B $(n=27)$ wurden nur quantitative endotracheale Aspiratkulturen vorgenommen. Als Grenzwerte für positive bakteriologische Kulturen wurden $10^{5}$ Keime pro $\mathrm{ml}$ für das endotracheale Aspirat, $10^{3}$ Keime pro ml für die PSB und $10^{4}$ Keime pro ml für die BAL verwandt.

Die initiale antibiotische Behandlung wurde bei 10 (42\%) der Patienten in der Gruppe A und bei 4 (16\%) der Patienten der Gruppe B verändert. Die Letalität in der Gruppe A betrug 46\% (11 von 24 Patienten) und 26\% (7 von 27 Patienten) in der Gruppe B. Die Autoren dieser Pilotstudie kamen zu dem Ergebnis, dass die bronchoskopischen Verfahren zwar zu häufigerem Antibiotikawechsel beitrugen, jedoch kein Einfluss auf einen letalen Verlauf hatten.

$\mathrm{Zu}$ einem anderen Ergebnis kommt eine kürzlich publizierte umfangreiche Studie an 413 Patienten in 31 französischen Intensivstationen [13]. Dabei wurden 204 Patienten bronchoskopisch untersucht, 209 Patienten wurden nur einer nicht quantitativen Analyse des endotrachealen Aspirates unterzogen. Als Endpunkte in der Studie wurden die Letalität, der Antibiotikagebrauch an den Tagen 14 und 28 auf der Intensivstation sowie die Anzahl der Organversagen gewählt. Bei Patienten mit einem invasiven 
bronchoskopischen Verfahren kam es zu einer statistisch signifikant geringeren Letalität am Tag 14 von $16,2 \%$ vs. $25,8 \%$ gegenüber den nur klinisch beurteilten Patienten. Auch der Sepsis-Score bezüglich Organversagen war signifikant niedriger am Tag 3 und Tag 7 bei den bronchoskopierten Patienten, die ebenfalls auch einen signifikant geringeren Antibiotikaverbrauch aufwiesen.

In der Multivarianzanalyse konnte eine signifikant niedrigere Letalität ebenfalls am Tag 28 bei den bronchoskopierten Patienten nachgewiesen werden. Die Autoren kommen zu der Folgerung, dass die invasive bronchoskopische Diagnostik bei Patienten mit dem Verdacht auf eine Beatmungspneumonie zu signifikant günstigeren Ergebnissen hinsichtlich der Letalität, der Häufigkeit des Organversagens sowie des Antibiotikaverbrauches beiträgt.

Unter Würdigung der dargestellten diagnostischen Ergebnisse und auch der zunehmenden Problemen von hochresistenten Erregern bei langzeitbeatmeten Patienten hat sich bei uns das in der Abb. 2 dargestellte Vorgehen zur adäquaten Diagnostik einer nosokomialen Pneumonie in den letzten Jahren sehr bewährt [26].

\section{Intensivmedizin}

\section{Resümee}

Nosokomiale Pneumonien sind zusammen mit der Sepsis die führende Ursache für einen infektionsbedingten Tod im Krankenhaus. Insbesondere auf Intensivstationen bei beatmeten Patienten ist die nosokomiale Pneumonie die häufigste Infektion und die Letalität bewegt sich zwischen $33 \%$ und $71 \%$. Verbrennungspatienten, Grunderkrankung wie Polytrauma sowie pulmonale und kardiale Vorerkrankungen disponieren signifikant mehr zu einer nosokomialen Pneumonie auf der Intensivstation.

In der Pathogenese der nosokomialen Pneumonie spielt die Kolonisation des Oropharynx und nachfolgend der Trachea mit gram-negativen Erregern die entscheidende Rolle und bestimmt daher auch das dominierende Erregermaterial. Neuerdings ist auch nachgewiesen worden, dass im unteren Drittel des Beatmungstubus ein Sekretfilm im Sinne eines Biomaterials gebildet wird mit entsprechender Persistenz der Erreger.

Die Unterteilung in eine frühe Pneumoniemanifestation bis zum 5. Tag des Krankenhausaufenthaltes im Vergleich zu der sogenannten späten Pneumonie ist sinnvoll, da mit unterschiedlichen Keimverteilungen zu rechnen ist.

In der Diagnostik der nosokomialen Pneumonie sollten vermehrt bronchoskopisch gestützte und quantitative mikrobiologische Verfahren eingesetzt werden mittels geschützter Bürste oder bronchoalveolärer Lavage. Zwar ist die Sensitivität und Spezifität dieser Verfahren noch nicht zufriedenstellend, jedoch kann mit diesen Maßnahmen offensichtlich die Letalität gesenkt, der Antibiotikaverbrauch vermindert und vor allem auch die Resistenz der jeweiligen Erreger exakt definiert werden.

\section{Fazit für die Praxis}

Die nosokomiale Pneumonie gehört neben den Harnwegsinfektionen zu den häufigsten krankenhauserworbenen Infektionen und stellt insbesondere auf Intensivstationen in Form der Beatmungspneumonie eine erhebliche diagnostische und therapeutische Herausforderung dar. Besonders gefährdet sind Verbrennungspatienten, Patienten mit Polytrauma sowie mit kardiopulmonalen Vorerkrankungen. Eine Unterteilung der frühen nosokomialen Pneumonie bis zum 5. Tag des Krankenhausaufenthaltes von der sogenannten späten Pneumoniemanifestation nach dem 5.-7. Tag im Hospital hat sich generell durchgesetzt. Diese Unterscheidung ist wegen der unterschiedlichen Erreger sinnvoll und sollte bei der empirischen Anfangstherapie berücksichtigt werden. In der Diagnostik sollten vermehrt bronchoskopische Verfahren (BAL, geschützte Bürste) eingesetzt werden, die mittels quantitativer mikrobiologischer Analyse bewertet werden sollten. Diese Verfahren können offensichtlich den Antibiotikaverbrauch vermindern, die Letalität senken und vor allem frühzeitig multiresistente Erreger nachweisen. 


\section{Literatur}

1. American Thoracic Society (1996) Hospitalacquired pneumonia in adults: Diagnosis, assessment of severity initial antimicrobial therapy and preventative strategies. Am J Respir Crit Care Med 153: 1711-1725

2. Baughman RP (2000) Protected-specimen brush technique in the diagnosis of ventilatorassociated pneumonia.

Chest 117 (Suppl): 203-206

3. Bowton DL (1999) Nosocomial pneumonia in the ICU: year 2000 and beyond. Chest 117 (Suppl): 28-33

4. Brown RB, Colodny SM, Drapkin MS (1995) One day prevalence study of 118 intensive care units (ICUs). Paper presented at: Fifth Annual Meeting of Healthcare Epidemiology of America, April 2-4, 1995 San Diego, CA

5. Chastre J, Fagon JY, Bornet M et al. (1995) Evaluation of bronchoscopic techniques for the diagnosis of nosocomial pneumonia. Am Rev Respir Dis 152: 231-240

6. Cook DJ, Walter SD, Cook RJ, et al. for the Canadian Critical Care Trials Group (1998) Incidence of and risk factors for ventilator-associated pneumonia in critically ill patients. Ann Intern Med 129: 433-440

7. Cook DJ, Gyatt G, Marshall J et al. for the Canadian Critical Care Trials Group (1998) A comparison of sucralfate and ranitidine for the prevention of upper gastrointestinal bleeding in patients requiring mechanical ventilation. N Engl J Med 338: 791-797

8. Cook D, Mandell L (2000) Endotracheal aspiration in the diagnosis of ventilator-associated pneumonia. Chest 117 (Suppl): 195-197

9. Doré P, Robert R, Grolhier G et al. (1996) Incidence of anaerobes in ventilator-assisted pneumonia with use of a protected specimen brush. Am J Resp Crit Care Med 253: 1292-1298

10. Drakulovic MB, Torres A, Bauer T et al. (1999) Supine body position as a risk factor for nosocomial pneumonia in mechanically ventilated patients: a randomised trial. Lancet 354: $1851-1858$
11. Ephgrave KS, Kleiman-Wexler R, Pfaller M et al. (1998) Effects of sucralfate versus antacids on gastric pathogens. Arch Surg 133: 251-257

12. Estes RJ, Meduri GU (1995) The pathogenesis of ventilator-associated pneumonia.I. Mechanisms of bacterial transcolonization and airway inoculation. Intensive Care Med 21: 365-83

13. Fagon JY et al. (2000) Invasive and noninvasive strategies for management of suspected ventilator-associated pneumonia. Ann Intern Med 132: 621-630

14. Feldman C, Kassel M, Cantrell J et al. (1999) The presence and sequence of endotracheal tube colonization in patients undergoing mechanical ventilation. Eur Respir J 13: 546-551

15. Francioli $\mathrm{P}$, Chastre J, Langer M, Santos J, Shah PM, Torres A (1997) Ventilator-associated pneumonia - understanding epidemiology and pathogenesis to guide prevention and empiric therapy. Clin Microb Infect 3 (Suppl): 61-73

16. George DL, Falk PS, Wunderink RG et al. (1998) Epidemiology of ventilator-acquired pneumonia based on protected bronchoscopic sampling. Am J Respir Crit Care Med 158: 1839-1847

17. Hauer $T$, Lacour M, Gastmeier P, Schulgen $G$, Schumacher M, Rüden H, Daschner F (1996) Nosokomiale Infektionen in Deutschland (NIDEP). Med Klinik 91: 681-686

18. Heyland DK, Cook DJ, Griffith L et al (1999) for the Canadian Critical Care Trial Group. The attributable morbidity and mortality of ventilatorassociated pneumonia in the critically ill patient. Am J Respir Crit Care Med 159: 1249-1256

19. Johanson WG, Higueth JH, Chaudhuri TR (1980) Bacterial adherence to epithelial cells in bacillary colonization of the respiratory tract. Am Rev Respir Dis 121: 56-63

20. Lode H, Schaberg T (1997) Therapie der nosokomialen Pneumonie. Dtsch Med Wochenschr 122: $93-96$

21. Lode H, Schaberg T, Raffenberg M, Mauch $H$ (1998) Nosocomial pneumonia in the critical care unit. Infect Crit Care 14: 119-133

22. Marquette CH, Copin MC, Wallet F et al. (1995) Ventilator-associated pneumonia: Prospective evaluation of the diagnostic yield of the protected specimen brush, endotracheal aspirates and bronchoalveolar lavage using the histopathological diagnosis as "gold standard". Am J Respir Crit Care Med 151: 1878-1888
23. Prod'hom G, Leuenberger P, Koerfer J et al. (1994) Nosocomial pneumonia in mechanically ventilated patients receiving antacid, ranitidine or sucralfate as prophylaxis for stress ulcer: a randomized controlled trial. Ann Intern Med 120: 653-662

24. Richards MJ, Edwards JR, Culver DH, Gaynes RP and the National Nosocomial Infections Surveillance System (1999) Nosocomial infections in medical intensive care units in the United States. Crit Care Med 27: 887-882

25. Sanchez-Nieto JM, Torres A, Garcia-Cordoba F et al. (1998) Impact of invasive and noninvasive quantitative culture sampling on outcome of ventilator-associated pneumonia. Am J Respir Crit Care Med 157: 371-376

26. Schaberg T, Lode H (1997) Diagnostik der nosokomialen Pneumonie. Dtsch Med Wochenschr 122: 61-64

27. Souweine B, Veber B, Bedos JP et al. (1998) Diagnostic accuracy of protected specimen brush and bronchoalveolar lavage in nosocomial pneumonia. impact of previous antimicrobial treatments. Crit Care Med 26: 236-244

28. Torres A, Aznar R, Gatell JM et al. (1990) Incidence, risk and prognosis factors of nosocomial pneumonia in mechanically ventilated patients. Am Rev Respir Dis 142: 523-528

29. Torres A, El-Ebiary M et al. (1994) Validation of different techniques for the diagnosis of ventilator-associated pneumonia. Am J Respir Crit Care Med 149: 324-331

30. Torres A, El-Ebiary M (2000) Bronchoscopic BAL in the diagnosis of ventilator associated pneumonia. Chest 117 (Suppl): 198-202

31. Vincent JL, Bihari DJ, Suter PM et al. (1995) The prevalence of nosocomial infection in intensive care units in Europe. JAMA 274: 639-614 